\title{
Matching of spatially homogeneous non-stationary space-times to vacuum in cylindrical symmetry
}

\author{
Paul $\operatorname{Tod}^{1} \&$ Filipe C. Mena ${ }^{1,2}$ \\ 1 Mathematical Institute, University of Oxford, St. Giles 24-29, Oxford OX1 3LB, U.K. \\ 2 Departamento de Matemática, Universidade do Minho, Gualtar, 4710 Braga, Portugal
}

10th May, 2004

\begin{abstract}
We study the matching of LRS spatially homogeneous collapsing dust space-times with non-stationary vacuum exteriors in cylindrical symmetry. Given an interior with diagonal metric we prove existence and uniqueness results for the exterior. The matched solutions contain trapped surfaces, singularities and Cauchy horizons. The solutions cannot be asymptotically flat and we present evidence that they are singular on the Cauchy horizons.
\end{abstract}

PACS: 04.20.Dw, 04.20.Ex, 04.20.Jb

Keywords: gravitational collapse; global models; cylindrical symmetry; black holes; trapped surfaces

\section{Introduction}

In cylindrical symmetry, unlike spherical symmetry, there exist exact solutions of the Einstein field equations (EFEs) which contain gravitational radiation. This raises the possibility of constructing collapsing matter cylinders matched to vacuum but radiating exteriors. In particular one can attempt to find analogues in cylindrical symmetry of the Oppenheimer-Snyder model. The Oppenheimer-Snyder model results from the matching of a ball in the Friedmann-LemaitreRobertson-Walker (FLRW) dust space-time to a Schwarzschild exterior across a timelike surface preserving the spherical symmetry. Thus there is no radiation in the exterior. This model cannot be generalised (in a physically reasonable way) to cylindrical symmetry with spatially homogeneous interiors and static exteriors. In fact, Mena, Tavakol and Vera [8] have shown that the matching of such space-times leads to very restrictive interior solutions with no evolution along one spacelike direction. As a next step one therefore considers a non-static exterior, but for simplicity retains a spatially homogeneous interior.

If such a model is to represent a cylinder of dust collapsing in a vacuum, then it should be asymptotically flat. Berger, Chrusciel and Moncrief [1] have shown that for cylindrically symmetric vacuum spacetimes the requirement of asymptotic flatness implies that the 2 -surfaces of transitivity are never trapped (specifically they show that one can choose a comoving radius of the form $R(T, \rho)=\rho$, which forbids trapping).

In this paper we investigate a family of models which result from the matching of a spatially homogeneous collapsing dust interior with a non-stationary vacuum exterior. These interiors always contain cylinders of symmetry (which we shall call just 'cylinders' for brevity) which are trapped near to the singularity. The marginally-trapped cylinders trace out a 3 -surface in the interior which eventually arrives at the boundary of the matter. Matching conditions at the boundary mean that the intersection 2-surface at the boundary is marginally-trapped as 
seen from the exterior and the results of Berger et al. [1] then prevent the exterior from being asymptotically flat. We shall argue that the failure of asymptotic flatness is in fact due to singularities in the vacuum exterior.

The technique will be to use the interior, which can be assumed to be known, to give data on the boundary of the matter, which will be a co-moving cylinder from the point of view of the interior but just a time-like surface made up of cylinders of transitivity seen from the outside. The EFEs in the exterior are a system of hyperbolic equations in the 2-dimensional quotient space $\mathcal{Q}$ of the space-time by the symmetries (this is the $(T, \rho)$-space) and data can be given on any non-characteristic curve in $\mathcal{Q}$. The boundary of the matter defines such a curve, even though it is a time-like surface in the full 4-metric. Therefore we can deduce existence and uniqueness of solutions in the domain of dependence in $\mathcal{Q}$ of the curve on which data is given. If the interior collapses to a singularity, as it does in these examples, then the data-curve has an end and there is a Cauchy horizon in the exterior. One expects the exterior to be singular on this Cauchy horizon, and we present some evidence for this belief. We shall interpret the singularity at the Cauchy horizon in terms of incoming gravitational radiation.

The plan of the paper is as follows. In Section 2 we review the matching procedure in general, and in Section 3 we introduce the interior and exterior metrics which we wish to match. The matching conditions for these metrics are written out in Section 4. In Section 5, we give some properties of the matched space-times when the metrics are diagonal in the canonical coordinates, obtaining existence from standard theorems for linear PDEs. We briefly discuss a non-diagonal example in Section 6 and finally in Section 7 we discuss some properties of the solutions, arguing that the Cauchy horizon is singular and giving a physical reason for this. Some equations are collected into an Appendix.

We use units such that $8 \pi G=c=1$. Greek indices take values 1 to 3 and latin indices 0 to 3 .

\section{Matching procedure in brief}

In this section, we recall how two space-times can be matched across a hypersurface by showing which junction conditions the respective metrics should satisfy. The first set of junction conditions ensures the continuity of the metrics across the matching hypersurface. The second set of junction conditions ensures the continuity of the second fundamental forms and, given the first set, is equivalent to requiring a non-singular Riemann tensor distribution, which prevents infinite discontinuities of matter and curvature across the matching hypersurface. In what follows we assume that the matching surface is non-null. For cases where the matching surfaces can change character see [7].

Let $\left(\mathcal{V}^{+}, g^{+}\right)$and $\left(\mathcal{V}^{-}, g^{-}\right)$be two $C^{3}$ space-times with oriented boundaries $\sigma^{+}$and $\sigma^{-}$, respectively, such that $\sigma^{+}$and $\sigma^{-}$are diffeomorphic. The matched space-time $(\mathcal{V}, g)$ is the disjoint union of $\mathcal{V}^{ \pm}$with the points in $\sigma^{ \pm}$identified such that the junction conditions are satisfied (Israel [4], Clarke \& Dray [3] and Mars \& Senovilla [7]). Since $\sigma^{ \pm}$are diffeomorphic, one can then view those boundaries as diffeomorphic to a 3-dimensional oriented manifold $\sigma$ which can be embedded in $\mathcal{V}^{+}$and $\mathcal{V}^{-}$. Let $\left\{\xi^{\alpha}\right\}$ and $\left\{x^{ \pm i}\right\}$ be coordinate systems on $\sigma$ and $\mathcal{V}^{ \pm}$ respectively. The two embeddings are given by the following $C^{3}$ maps

$$
\begin{aligned}
\Phi^{ \pm}: \sigma & \longrightarrow \mathcal{V}^{ \pm} \\
\xi^{\alpha} & \mapsto x^{i^{ \pm}}=\Phi^{i^{ \pm}}\left(\xi^{\alpha}\right),
\end{aligned}
$$

such that $\sigma^{ \pm} \equiv \Phi^{ \pm}(\sigma) \subset \mathcal{V}^{ \pm}$. The diffeomorphism from $\sigma^{+}$to $\sigma^{-}$is $\Phi^{-} \circ \Phi^{+-1}$. 
Given the basis $\left\{\partial /\left.\partial \xi^{\alpha}\right|_{p}\right\}$ of the tangent plane $T_{p} \sigma$ at some $p \in \sigma$, the push-forwards $\left.d \Phi^{ \pm}\right|_{p}$ map $\left\{\partial /\left.\partial \xi^{\alpha}\right|_{p}\right\}$ into three linearly independent vectors at $\Phi^{ \pm}(p)$ represented by $\left.\vec{e}_{\alpha}^{ \pm}\right|_{\Phi^{ \pm}(p)}$ :

$$
d \Phi^{ \pm}\left(\left.\frac{\partial}{\partial \xi^{\alpha}}\right|_{\sigma}\right)=\left.\left.\frac{\partial \Phi^{ \pm i}}{\partial \xi^{\alpha}} \frac{\partial}{\partial x^{ \pm i}}\right|_{\sigma^{ \pm}} \equiv \vec{e}_{\alpha}^{ \pm}\right|_{\sigma^{ \pm}}=\left.e_{\alpha}^{ \pm i} \frac{\partial}{\partial x^{ \pm i}}\right|_{\sigma^{ \pm}} .
$$

On the other hand, using the pull-backs $\Phi^{ \pm *}$ of the maps $\Phi^{ \pm}$, the metrics $g^{ \pm}$can be mapped to $\sigma$ given two symmetric 2-covariant tensors $\bar{g}^{+}$and $\bar{g}^{-}$whose components in the basis $\left\{d \xi^{\alpha}\right\}$ are

$$
\left.\bar{g}_{\alpha \beta}^{ \pm} \equiv e_{\alpha}^{ \pm i} e_{\beta}^{ \pm j} g_{i j}\right|_{\sigma^{ \pm}}=\left.\left(\vec{e}_{\alpha}^{ \pm} \cdot \vec{e}_{\beta}^{ \pm}\right)\right|_{\sigma^{ \pm}} .
$$

The first matching conditions are given by the equality of the first fundamental forms (Israel [4])

$$
\bar{g}_{\alpha \beta}^{+}=\bar{g}_{\alpha \beta}^{-} .
$$

We note that the existence of a continuous metric allows for the treatment of Einstein's field equations in the distributional sense (see e.g. Mars \& Senovilla [7]). The bases $\left\{\left.\vec{e}_{\alpha}^{+}\right|_{p}\right\}$ and $\left\{\left.\vec{e}_{\alpha}^{-}\right|_{p}\right\}$ can be identified,

$$
d \Phi^{+}\left(\left.\frac{\partial}{\partial \xi^{\alpha}}\right|_{\sigma}\right)=d \Phi^{-}\left(\left.\frac{\partial}{\partial \xi^{\alpha}}\right|_{\sigma}\right)
$$

as can the hypersurfaces $\sigma^{+} \equiv \sigma^{-}$, so henceforth we represent both $\sigma^{ \pm}$by $\sigma$.

We now define a 1 -form $\boldsymbol{n}$, normal to the hypersurface $\sigma$, as

$$
\boldsymbol{n}^{ \pm}\left(\vec{e}_{\alpha}^{ \pm}\right)=0
$$

The vectors $\left\{\vec{n}^{ \pm}, \vec{e}_{\alpha}^{ \pm}\right\}$constitute a basis on the tangent spaces to $\mathcal{V}^{ \pm}$at $\sigma^{ \pm}$. Since the first junction conditions allows the identification of $\left\{\vec{e}_{\alpha}^{+}\right\}$with $\left\{\vec{e}_{\alpha}^{-}\right\}$, we only have to ensure that both bases have the same orientation and that $n_{i}^{+} n^{+i} \stackrel{\sigma}{=} n_{i}^{-} n^{-i}$ is satisfied in order to identify the whole 4-dimensional tangent spaces of $\mathcal{V}^{ \pm}$at $\sigma,\left\{\vec{n}^{+}, \vec{e}_{\alpha}^{+}\right\} \equiv\left\{\vec{n}^{-}, \vec{e}_{\alpha}^{-}\right\}$.

The second fundamental forms are given by

$$
H_{\alpha \beta}^{ \pm}=-n_{i}^{ \pm} e_{\alpha}^{ \pm j} \nabla_{j}^{ \pm} e_{\beta}^{ \pm i}
$$

and the second matching conditions, for non-null surfaces, are the equality of the second fundamental forms

$$
H_{\alpha \beta}^{+}=H_{\alpha \beta}^{-} \text {. }
$$

We note that these matching conditions do not depend on the choice of the normal vectors.

\section{Cylindrically symmetric exterior and spatially homogeneous interior}

We are interested in the particular cases for which the matching surface inherits a certain symmetry of the two space-times $\left(\mathcal{V}^{ \pm}, g^{ \pm}\right)$. Such matching is said to preserve the symmetry. In practice one demands that the matching hypersurface is tangent to the orbits of the symmetry group to be preserved (see e.g. [11]). In the case we shall consider below, the symmetry group is an Abelian $G_{2}$ and we are interested in the matching of a cylindrical symmetric exterior to a spatially homogeneous (and anisotropic) interior preserving the $G_{2}$ symmetry.

Since we want to preserve the symmetry of a $G_{2}$ exterior space-time we must ensure that the interior also has the same group of symmetries, possibly as a subgroup of a larger isometry group. We wish to preserve an axial symmetry so that we require the interior to have an axial 
symmetry in the sense of a periodic Killing vector, vanishing on a symmetry axis. In the exterior $\mathcal{V}^{+}$the Killing vectors commute, so we require this in the interior. Thus we require the interior to be spatially homogeneous, with an axial symmetry and at least one Killing vector commuting with the axial symmetry. This is sufficient to force the interior to be an LRS space-time (see Mena, Tavakol \& Vera [8]).

We deal separately the metric forms for the interior and exterior.

\subsection{Interior}

All the LRS spatially homogeneous metrics can be written in the compact form

$$
d s^{2-}=-d t^{2}+a(t)^{2} \boldsymbol{\theta}^{2}+b(t)^{2}\left[(d r-\epsilon r d z)^{2}+\Sigma(r)^{2} d \varphi^{2}\right]
$$

where

$$
\boldsymbol{\theta}=d z+n(F(r)+k) d \varphi
$$

and the functions $\Sigma$ and $F$ are given by

$$
\Sigma(r)=\left\{\begin{array}{cl}
\sin r, & k=+1 \\
r, & k=0 \\
\sinh r, & k=-1
\end{array} \quad \text { and } \quad F(r)=\left\{\begin{array}{cl}
-\cos r, & k=+1 \\
r^{2} / 2, & k=0 \\
\cosh r, & k=-1
\end{array}\right.\right.
$$

and where $\epsilon$ and $n$ are given such that

$$
\epsilon=0,1 ; \quad n=0,1 ; \quad \epsilon n=\epsilon k=0 .
$$

We note that

$$
\Sigma=F_{r} ; \quad\left(\Sigma_{r}\right)^{2}+k \Sigma^{2}=1,
$$

where a subscript denotes the partial derivative with respect to the indicated variable. The axial Killing vector is then given by

$$
\vec{\eta}_{1}=\partial_{\varphi}
$$

while the other three Killing vectors $\vec{\eta}_{i}, i=2$ to 4 are

$$
\begin{aligned}
& \vec{\eta}_{2}=\partial_{z} \\
& \vec{\eta}_{3}=\sin \varphi e^{\epsilon z} \partial_{r}+\cos \varphi\left(e^{\epsilon z} f(r) \partial_{\varphi}+g(r) \partial_{z}\right), \\
& \vec{\eta}_{4}=\cos \varphi e^{\epsilon z} \partial_{r}-\sin \varphi\left(e^{\epsilon z} f(r) \partial_{\varphi}+g(r) \partial_{z}\right)
\end{aligned}
$$

where we have defined $f(r)=\Sigma_{r} / \Sigma$ and $g(r)=n(\Sigma-f(F+k))$. The Killing vector which commutes with the axial Killing vector $\vec{\eta}_{1}$ is $\vec{\eta}_{2}$. We now assume a perfect fluid interior and recall a result which is a direct consequence of the so-called Israel conditions $n^{-i} T_{i j}^{-} \stackrel{\sigma}{=} n^{+i} T_{i j}^{+}$:

Lemma 1 Let $(\mathcal{V}, g)$ be a space-time resulting from the matching of two space-times $\left(\mathcal{V}^{ \pm}, g^{ \pm}\right)$. If $\left(\mathcal{V}^{-}, g^{-}\right)$has a perfect fluid and $\left(\mathcal{V}^{+}, g^{+}\right)$vacuum then the perfect fluid has $p \stackrel{\sigma}{=} 0$. Furthermore, if $\left(\mathcal{V}^{-}, g^{-}\right)$is spatially homogeneous then $p=0$ everywhere.

The metric for the interior will be assumed to be given by (4) with dust as matter content. For convenience we shall use the form

$$
d s^{2-}=-A^{2} d t^{2}+B^{2} d r^{2}-2 \epsilon r B^{2} d r d z+C^{2} d \varphi^{2}+2 E d z d \varphi+D^{2} d z^{2},
$$




\begin{tabular}{|c|c|c|c|}
\hline KS/Bianchi types & $\epsilon$ & $n$ & $k$ \\
\hline I & 0 & 0 & 0 \\
KS & 0 & 0 & 1 \\
KS,III & 0 & 0 & -1 \\
IX & 0 & 1 & 1 \\
II & 0 & 1 & 0 \\
VIII,III $_{\text {V,VII }_{h}}$ & 0 & 1 & -1 \\
& 1 & 0 & 0 \\
\hline
\end{tabular}

Table 1: Classification of the possible $G_{3}$ on $S_{3}$ subgroups according to the values of $\{\epsilon, k, n\}$ for the metric given by (4). KS denotes Kantowski-Sachs.

where $A, B, C, D$ and $E$ functions of $t$ and $r$. The line-element (4) is recovered by making the identifications

$$
\begin{aligned}
A^{2}(t, r) & =1 \\
B^{2}(t, r) & =b^{2}(t), \\
C^{2}(t, r) & =b^{2}(t) \Sigma^{2}(r, k)+n a^{2}(t)(F(r, k)+k)^{2}, \\
D^{2}(t, r) & =a^{2}(t)+\epsilon r^{2} b^{2}(t), \\
E(t, r) & =n a^{2}(t)(F(r, k)+k) .
\end{aligned}
$$

In the following we shall take the functions in (6) to be arbitrary functions, with

$$
\epsilon E=0
$$

which follows from (5).

\subsection{Exterior}

The exterior will be assumed to be cylindrically symmetric vacuum (with an Abelian $G_{2}$ on $S_{2}$ ). The most general metric form can be written as [1]

$$
d s^{2+}=e^{2(\gamma-\psi)}\left(-d T^{2}+d \rho^{2}\right)+R^{2} e^{-2 \psi} d \tilde{\varphi}^{2}+e^{2 \psi}(d \tilde{z}+W d \tilde{\varphi})^{2}
$$

where $\psi, \gamma, R, W$ are functions of the coordinates $\rho, T$. For $W \neq 0$ the two Killing vectors are not hypersurface orthogonal and the cylindrical gravitational waves have two polarisations states. One of the EFEs is (33)

$$
R_{T T}-R_{\rho \rho}=0
$$

which has the general solution

$$
R(T, \rho)=F(x)+G(y)
$$

where

$$
x=T+\rho ; y=T-\rho .
$$

The metric (7) is invariant under a coordinate transformation

$$
\tau:(x, y) \rightarrow(f(x), g(y)),
$$

where $f$ and $g$ are arbitrary differentiable functions (with non-zero derivative). One may use this freedom to restrict $R$, but we shall make a different choice below, which is to use $\tau$ to 
prescribe the matching surface $\sigma^{+}$. The choices $W=0$ and $R=\rho$ in (7) lead to the well-known Einstein-Rosen metric.

It will be important below to see when the 2-surfaces of transitivity are trapped or marginallytrapped. From (7) we see that these 2 -surfaces have intrinsic metric $R d \tilde{\varphi} d \tilde{z}$. Taking the two null-normals to these 2-surfaces, we see that such a 2 -surface is trapped if

$$
4 R_{x} R_{y}=\left(R_{T}-R_{\rho}\right)\left(R_{T}+R_{\rho}\right) \geq 0
$$

and is marginally-trapped if this expression is exactly zero. It follows from (8) that if $R_{x}=0$, at say $x=x_{0}$, then all 2 -surfaces of transitivity with $x=x_{0}$ are marginally-trapped. Similar statements follow with $y$ replacing $x$.

\subsection{The matching hypersurfaces}

The embeddings $\sigma^{ \pm}$can be defined by using coordinates on $\sigma$ denoted by $\xi^{\alpha}=\{\lambda, \phi, \zeta\}$. We choose $\phi$ such that

$$
d \Phi^{-}\left(\frac{\partial}{\partial \phi}\right)=\left.\frac{\partial}{\partial \varphi}\right|_{\sigma^{-}}=\vec{e}_{2}^{-}
$$

Since we perform the matching preserving the $G_{2}$ symmetry there must a vector field $\vec{\gamma}$ which together with $\partial /\left.\partial \varphi\right|_{\sigma^{-}}$generates the $G_{2}$ on $S_{2}$. In particular $\vec{\gamma}$ must satisfy

$$
d \Phi^{-}(\vec{\gamma})=\left.c_{1} \frac{\partial}{\partial \varphi}\right|_{\sigma^{-}}+\left.c_{2} \frac{\partial}{\partial z}\right|_{\sigma^{-}}
$$

with $c_{1}, c_{2}$ constants such that $c_{2} \neq 0$ and otherwise arbitrary. We now use the fact that $\partial / \partial \phi$ and $\vec{\gamma}$ must commute to choose $\vec{\gamma}=\partial / \partial \zeta$ and a coordinate transformation $\zeta^{\prime}=c_{2} \zeta, \phi^{\prime}=\phi+c_{1} \zeta$ to get (after dropping the primes):

$$
d \Phi^{-}\left(\frac{\partial}{\partial \zeta}\right)=\left.\frac{\partial}{\partial z}\right|_{\sigma^{-}}=\vec{e}_{3}^{-}
$$

Finally, $\lambda$ is chosen such that $d \Phi^{-}(\partial / \partial \lambda)$ is orthogonal to $\vec{e}_{2}^{-}$and $\vec{e}_{3}{ }^{-}$. This implies

$$
d \Phi^{-}\left(\frac{\partial}{\partial \lambda}\right)=\left.\frac{\partial \Phi^{0-}}{\partial \lambda} \frac{\partial}{\partial t}\right|_{\sigma^{-}}+\left.\frac{\partial \Phi^{1-}}{\partial \lambda} \frac{\partial}{\partial r}\right|_{\sigma^{-}}+\left.\epsilon \Phi^{1-} \frac{B^{2}}{D^{2}} \frac{\partial \Phi^{1-}}{\partial \lambda} \frac{\partial}{\partial z}\right|_{\sigma^{-}}=\vec{e}_{1}^{-}
$$

By denoting the embedding $\left\{\Phi^{0-}, \Phi^{1-}, \Phi^{2-}, \Phi^{3-}\right\}$ in $(1)$ as $\{t, r, \varphi, z\}$, the matching surface $\sigma^{-}$ is parametrized as

$$
\sigma^{-}=\left\{(t, r, \varphi, z): t=t(\lambda), r=r(\lambda), \varphi=\phi, z=\zeta+f_{z}(\lambda)\right\},
$$

where $t(\lambda)$ and $r(\lambda)$ are functions of $\lambda$ restricted by the fact that $d \Phi^{-}$has to be of rank 3 , that is $\dot{t}^{2}+\dot{r}^{2} \neq 0$, and

$$
\dot{f}_{z}(\lambda) \stackrel{\sigma}{=} \epsilon r(\lambda) \dot{r}(\lambda) \frac{B^{2}}{D^{2}}
$$

where the dot denotes differentiation with respect to $\lambda$.

Consider now the embedding $\Phi^{+}$. The axial killing vectors from both $\sigma^{ \pm}$must coincide at the matching surface so

$$
d \Phi^{+}\left(\frac{\partial}{\partial \phi}\right)=\left.\frac{\partial}{\partial \tilde{\varphi}}\right|_{\sigma^{+}}=\vec{e}_{2}^{+}
$$


and $\left.d \Phi^{+}(\partial / \partial \zeta)\right|_{\sigma^{+}}$must complete the basis of the $G_{2}$ on $S_{2}$. By a similar procedure as for $\sigma^{-}$ we can always choose $\zeta$ and $\lambda$ such that

$$
\begin{aligned}
d \Phi^{+}\left(\frac{\partial}{\partial \phi}\right) & =\left.\frac{\partial}{\partial \zeta}\right|_{\sigma^{+}}=\vec{e}_{3}^{+} \\
d \Phi^{+}\left(\frac{\partial}{\partial \lambda}\right) & =\left.\frac{\partial \Phi^{0+}}{\partial \lambda} \frac{\partial}{\partial T}\right|_{\sigma^{+}}+\left.\frac{\partial \Phi^{1+}}{\partial \lambda} \frac{\partial}{\partial \rho}\right|_{\sigma^{+}}=\vec{e}_{1}^{+}
\end{aligned}
$$

so that matching surface $\sigma^{+}$can be parametrized by

$$
\sigma^{+}=\{(T, \rho, \tilde{\varphi}, \tilde{z}): T=T(\lambda), \rho=\rho(\lambda), \tilde{\varphi}=\phi, \tilde{z}=\zeta\}
$$

\section{The matching conditions}

In order to derive the junction conditions we have to calculate the first and second fundamental forms for both $\sigma^{+}$and $\sigma^{-}$. For the $g^{-}$metric, the parametric form of $\sigma^{-}$gives $\left.d t\right|_{\sigma^{-}}=\dot{t} d \lambda,\left.d r\right|_{\sigma^{-}}=\dot{r} d \lambda,\left.d \varphi\right|_{\sigma^{-}}=d \phi$ and $\left.d z\right|_{\sigma^{-}}=d \zeta+\dot{f}_{z} d \lambda$. Using (11), the first fundamental form $\bar{g}_{a b}^{-}$on $\sigma^{-}$can be written as

$$
\left.d s^{2-}\right|_{\sigma^{-}} \stackrel{\sigma}{=}\left(-A^{2} \dot{t}^{2}+\mathcal{B}^{2} \dot{r}^{2}\right) d \lambda^{2}+C^{2} d \phi^{2}+2 E d \phi d \zeta+D^{2} d \zeta^{2},
$$

where

$$
\mathcal{B}^{2} \equiv B^{2}\left(1-\epsilon r^{2} \frac{B^{2}}{D}\right),
$$

Similarly the first fundamental form $\bar{g}^{+}$on $\sigma^{+}$is given by

$$
\left.d s^{2+}\right|_{\sigma^{+}} \stackrel{\sigma}{=} e^{2(\gamma-\psi)}\left(-\dot{T}^{2}+\dot{\rho}^{2}\right) d \lambda^{2}+\left(R^{2} e^{-2 \psi}+W^{2} e^{2 \psi}\right) d \phi^{2}+2 W e^{2 \psi} d \zeta d \phi+e^{2 \psi} d \zeta^{2}
$$

and then the first set of matching conditions (2) is:

$$
\begin{array}{rll}
-A^{2} \dot{t}^{2}+\mathcal{B}^{2} \dot{r}^{2} & \stackrel{\sigma}{=} & e^{2(\gamma-\psi)}\left(-\dot{T}^{2}+\dot{\rho}^{2}\right) \\
C^{2} & \stackrel{\sigma}{=} & R^{2} e^{-2 \psi}+W^{2} e^{2 \psi} \\
D^{2} & \stackrel{\underline{\sigma}}{=} & e^{2 \psi} \\
E & \stackrel{\sigma}{=} & W e^{2 \psi}
\end{array}
$$

The normal forms to the matching surfaces can be taken as

$$
\begin{aligned}
& \boldsymbol{n}^{-}=\left.A \mathcal{B}(-\dot{r} d t+\dot{t} d r)\right|_{\sigma^{-}} \\
& \boldsymbol{n}^{+}=\left.e^{2(\gamma-\psi)}(-\dot{\rho} d T+\dot{T} d \rho)\right|_{\sigma^{+}}
\end{aligned}
$$

In our case, the interior space-time contains dust and so the matching is performed across a time-like hypersurface ruled by matter trajectories, which are geodesics (this follows from the matching conditions). Therefore, $\dot{t}=1$ and $\dot{r}=0$. From the point of view of the exterior, the matching surface is given parametrically as $(x(\lambda), y(\lambda))$ in terms of the coordinates $x, y$ in $(9)$. The product $\dot{x} \dot{y}$ does not vanish since the matching surface is everywhere time-like and so we may exploit the transformation $\tau$ of (9) to set $x=\lambda, y=\lambda$, or equivalently $\dot{T}=1$ and $\dot{\rho}=0$. Now the matching surface is at $r=r_{0}, \rho=\rho_{0}$ and the time-coordinates can be taken to agree, $t=T$. 
Under these circumstances we get the first set of matching conditions (12)-(15) in the form:

$$
\begin{array}{rll}
\gamma & \stackrel{\sigma}{=} & \psi \\
b^{2} \Sigma^{2}+n a^{2}(F+k)^{2} & \stackrel{\sigma}{=} & R^{2} e^{-2 \psi}+W^{2} e^{2 \psi} \\
a^{2}+\epsilon r^{2} b^{2} & \stackrel{\sigma}{=} & e^{2 \psi} \\
n a^{2}(F+k) & \stackrel{\sigma}{=} & W e^{2 \psi}
\end{array}
$$

and the second set of matching conditions (3) as

$$
\begin{array}{rll}
\psi_{\rho} & \stackrel{\sigma}{=} & 0 \\
\gamma_{\rho} & \stackrel{\sigma}{=} & 0 \\
\frac{n a^{2} F_{r}}{b} & \stackrel{\sigma}{=} & e^{2 \psi} W_{\rho} \\
\frac{C C_{r}}{b} & \stackrel{\sigma}{=} & R R_{\rho} e^{-2 \psi}+W W_{\rho} e^{2 \psi},
\end{array}
$$

and for $(D B)_{t} \neq 0$

$$
\epsilon \stackrel{\sigma}{=} 0
$$

As a consequence of (16) and $\dot{T}=1$ we also have

$$
\gamma_{T} \stackrel{\sigma}{=} \psi_{T}
$$

The fact that $\epsilon=0$ excludes some Bianchi types:

Proposition $1 A G_{4}$ on $S_{3} L R S$ spacetime admitting a simply transitive subgroup $G_{3}$ of Bianchi types $V$ or $V I I_{h}$ cannot be matched to a cylindrically symmetric non-stationary vacuum spacetime across timelike surfaces preserving the cylindrical symmetry.

We shall now look more closely at the cases with diagonal metrics.

\section{Diagonal cases}

For simplicity, we begin with a diagonal interior metric, so that $\epsilon=n=0$. Now $W$ and $W_{\rho}$ vanish on $\sigma$, from (19) and (22). In each case below we first establish that $R \neq 0$ in the exterior, and we can then deduce that $W=0$ in the exterior, from uniqueness for the linear equation (35) which it satisfies. Assuming this for now, the matching conditions give

$$
\begin{array}{rll}
e^{\psi} & \stackrel{\sigma}{=} & a \\
R & \stackrel{\sigma}{=} & a b \Sigma \\
R_{\rho} & \stackrel{\sigma}{=} & a \Sigma_{r},
\end{array}
$$

together with $\gamma \stackrel{\sigma}{=} \psi$ and $\gamma_{\rho} \stackrel{\sigma}{=} \psi_{\rho}=0$. We first consider the two explicit solutions corresponding to the flat $(k=0)$ FLRW and to the Bianchi I cases and then consider implicit solutions for Kantowski-Sachs and Bianchi III models. We may summarise the results that we shall find in the following theorem.

Theorem 1 A cylindrical interior dust metric constructed from a collapsing FLRW, Bianchi I or Kantowski-Sachs cosmological model can be matched to a diagonal vacuum exterior within the domain of dependence $\mathcal{D}$ of the matching surface. The metric is smooth up to the Cauchy horizon $\mathcal{H}$, but there are trapped surfaces in the exterior. If the interior is a Bianchi III model 
(with space sections $\mathbb{R} \times S^{2}$ ) then the matching can be performed as far as the Cauchy horizons of the past and future singularities provided the matter occupies less than half of the $S^{2}$. If the matter occupies more than half of the $S^{2}$ then the matching is at least possible near to the matching surface.

In Section 7 we shall present evidence that the exterior is in fact singular on the Cauchy surface, and we shall suggest a physical origin for this singularity.

\section{1 $\quad$ FLRW $k=0$}

We want the interior metric to be collapsing so that the relevant solution to EFEs (38) is explicitly given by

$$
a(t)=b(t)=(\alpha-t)^{2 / 3},
$$

for $t \in(-\infty, \alpha)$. The matching surface $\sigma$ is the cylinder $\rho=\rho_{0}, T<\alpha$, terminating in the singularity at $T=\alpha$. At $\sigma$, by (25) and (26) we have

$$
\begin{aligned}
& R \stackrel{\sigma}{=} r_{0}(\alpha-T)^{4 / 3} \\
& R_{\rho} \stackrel{\sigma}{=}(\alpha-T)^{\frac{2}{3}} \text {, }
\end{aligned}
$$

so that in particular $R$ and $R_{\rho}$ on $\sigma$ are positive for $T<\alpha$, vanishing only at $T=\alpha$.

We shall determine the exterior using the matching conditions, in the domain of dependence $\mathcal{D}=\left\{T+\rho<\alpha+\rho_{0}, \rho \geq \rho_{0}\right\}$ of $\sigma$ in $\mathcal{Q}$, when $\sigma$ is taken to be at $\rho=\rho_{0}$. The boundary of the domain of dependence, at $T+\rho=\alpha+\rho_{0}$, is the Cauchy horizon which we shall call $\mathcal{H}$. It follows from the D'Alembert solution of the one-dimensional wave equation for $R$ that, since the data for it is positive, $R$ is positive in $\mathcal{D}$. (Now we may conclude that $W=0$ ). We may obtain $R$ explicitly from (28), (29) and (8) as

$$
R=\frac{r_{0}}{2}\left(\alpha+\rho_{0}-T-\rho\right)^{\frac{4}{3}}-\frac{3}{10}\left(\alpha+\rho_{0}-T-\rho\right)^{\frac{5}{3}}+\frac{r_{0}}{2}\left(\alpha+\rho_{0}-T+\rho\right)^{\frac{4}{3}}+\frac{3}{10}\left(\alpha+\rho_{0}-T+\rho\right)^{\frac{5}{3}} .
$$

From this formula for $R$ we can investigate the condition (10) for trapped-ness. On $\sigma$ it is easy to see that $R_{y}$ vanishes only at $T=\alpha$, while $R_{x}$ vanishes at $T=\alpha$ and at $T:=T_{0}=\alpha-\left(\frac{8 r_{0}}{3}\right)^{3 / 2}$. Thus there is a marginally-trapped cylinder at $T=T_{0}$ on $\sigma$ and this gives rise in the exterior to whole null hypersurface $\mathcal{N}_{0}$ of marginally-trapped cylinders at $T+\rho=T_{0}+\rho_{0}$. Cylinders to the past of $\mathcal{N}_{0}$ are not trapped and those to the future are.

We have seen that $R$ is non-negative in $\mathcal{D}$ and by inspection it vanishes only in the corner at $T=\alpha, \rho=\rho_{0}$, and is smooth in the interior of $\mathcal{D}$, though not along the Cauchy horizon $\mathcal{H}$ at $T+\rho=\alpha+\rho_{0}$ where second and higher derivatives diverge.

At $\sigma$, by (20), (24) and (27) we have

$$
\begin{aligned}
& \psi \stackrel{\sigma}{=} \frac{2}{3} \ln (\alpha-T) \\
& \psi_{\rho} \stackrel{\sigma}{=} 0 \text {. }
\end{aligned}
$$

This is the data for $\psi$ in the exterior, and it is smooth (in fact analytic) up to $T=\alpha$. Given what we know about $R$, it now follows from standard theorems for linear hyperbolic equations (see e.g. section 6.5 in [10]) that a unique $\psi$ exists satisying (34) in the interior of $\mathcal{D}$, with the given data on $\sigma$. There is no reason to expect the solution to be well-defined at $\mathcal{H}$, since it diverges on $\sigma$ at $T=\alpha$, and in fact we shall argue in section 7 that there is a curvature singularity along $\mathcal{H}$. 
Since $\gamma \stackrel{\sigma}{=} \psi$ and $\gamma_{\rho} \stackrel{\sigma}{=} \psi_{\rho}$, once we have $\psi$ in $\mathcal{D}$ the same argument applied to (32) gives existence and uniqueness for $\gamma$ in $\mathcal{D}$, and we have constructed a vacuum exterior which matches on to the dust interior, at least inside $\mathcal{D}$. (In this and all the examples, the constraint equations (36) and (37) are identically satisfied by virtue of the field equations in the interior, as must be the case.)

\subsection{Bianchi I}

The collapsing solution to the EFEs (38) in the interior can be taken to be

$$
\begin{aligned}
& a(t)=(\alpha-t)^{-1 / 3}(\beta-t) \\
& b(t)=(\alpha-t)^{2 / 3}
\end{aligned}
$$

for $t<\min \{\alpha, \beta\}$ and $r \leq r_{0}$. If $\alpha=\beta$ this reduces to the previous case. The character of the solution depends on which is the smaller of $\alpha$ and $\beta$. If $\alpha<\beta$ then $a$ diverges and $b$ vanishes at the singularity. This is the behaviour of the Kasner (vacuum) solution and we can call it Kasner-like. If $\alpha=\beta$ then $a$ and $b$ vanish at the same rate, which is precisely the FLRW behaviour so that we may call it FLRW-like. If $\alpha>\beta$ then $a$ vanishes but $b$ does not and we call this disk-like.

Since the interior is given explicitly we can use the matching conditions as before to get the exterior functions. We again find that the data $R$ and $R_{\rho}$ are positive so that $R$ is positive in $\mathcal{D}$ and $W=0$. Explicitly, (25), (26), (8) imply

$$
\begin{aligned}
R= & \frac{r_{0}}{2}\left(\alpha+\rho_{0}-T-\rho\right)^{\frac{1}{3}}\left(\beta+\rho_{0}-T-\rho\right)-\frac{3}{4}\left(\alpha+\rho_{0}-T-\rho\right)^{\frac{2}{3}}\left(\beta+\rho_{0}-T-\rho\right) \\
& +\frac{9}{20}\left(\alpha+\rho_{0}-T-\rho\right)^{\frac{5}{3}} \\
& +\frac{r_{0}}{2}\left(\alpha-\rho_{0}-T+\rho\right)^{\frac{1}{3}}\left(\beta-\rho_{0}-T+\rho\right)+\frac{3}{4}\left(\alpha-\rho_{0}-T+\rho\right)^{\frac{2}{3}}\left(\beta-\rho_{0}-T+\rho\right) \\
& -\frac{9}{20}\left(\alpha+\rho_{0}-T+\rho\right)^{\frac{5}{3}} .
\end{aligned}
$$

It is straightforward to see that $R_{y}$ is always negative on $\sigma$ while $R_{x}$ necessarily changes sign. Thus, by (10), there is always a marginally-trapped cylinder on $\sigma$ and this gives rise to a null hypersurface of marginally-trapped cylinders $\mathcal{N}_{0}$ as in the previous case (for suitable combinations of the parameters $\alpha, \beta$ and $\rho_{0}$ there may be three such hypersurfaces). However $R$ is positive in the exterior, vanishing only in the corner $\rho=\rho_{0}, T=\min (\alpha, \beta)$. Note also that $R_{x}$ diverges at the singularity, and therefore on the Cauchy horizon, if the singularity is Kasner-like.

For the other exterior metric functions we get from (24)

$$
\psi \stackrel{\sigma}{=} \gamma \stackrel{\sigma}{=} \ln (\beta-T)-\frac{1}{3} \ln (\alpha-T)
$$

and with $\gamma_{\rho} \stackrel{\sigma}{=} \psi_{\rho} \stackrel{\sigma}{=} 0$ we again have existence and uniqueness of solutions in the interior of $\mathcal{D}$. As before, we expect the solution to diverge near $\mathcal{H}$, a point we shall return to below. We have then constructed a vacuum exterior which matches on to the Bianchi I dust interior, at least inside $\mathcal{D}$.

\subsection{Kantowski-Sachs and Bianchi III}

For these interiors, implicit solutions are known and we give them in the appendix. Dealing first with the Kantowski-Sachs metric, the data for $R$ are positive on $\sigma$ so that as usual $R$ is 
positive in the exterior, out to the Cauchy horizon, and $W=0$. The singularity is Kasner-like, FLRW-like or disk-like according as $C_{0}$ is positive, zero or negative in (40). In each case, the quantity $R_{T}-R_{\rho}$ changes sign from positive in the infinite past to negative near the singularity so that there are trapped cylinders on $\sigma$ near the singularity and these persist in the exterior as before. The existence argument carries through just as before, out to the Cauchy horizon.

There are two extra complications with the Bianchi III metric. The first is that there are singularities in the past as well as in the future. The second is that $\Sigma=\sin r$ so that $R_{\rho} \stackrel{\sigma}{=} a \cos r_{0}$ which can be negative or zero.

Suppose the past and future singularities lie on $\sigma$ at $T=T_{0}$ and $T=T_{1}$ respectively, then the domain of dependence is restricted to

$$
\mathcal{D}=\left\{T+\rho<T_{1}+\rho_{0}, T-\rho>T_{0}-\rho_{0}\right\}
$$

and there is a past Cauchy horizon as well as a future one. The nature of the singularities depends on the relative sizes of the constants $M$ and $C_{0}$ in (39) and various combinations of Kasner-like, disk-like and FLRW-like are possible. However, at least one of the past and future singularities is always Kasner-like.

Now for $R$, suppose first that $0<r_{0} \leq \pi / 2$, then the data for $R$ are positive so $R$ is positive in the exterior and $W=0$. The proof of existence is as before. On the other hand, if $\pi / 2<r_{0}<\pi$ then $R_{\rho}$ is negative on $\sigma$ and so $R$ may vanish inside $\mathcal{D}$. If $R$ vanished in a smooth way, this could just be the sign of a (second) regular axis for the axial Killing vector. To see that this is not the case we recall from the discussion of the Bianchi I case above that the derivative of $R$ is singular on the Cauchy horizon emanating from a Kasner-like singularity. Since one of the singularities must be Kasner-like, $R$ cannot be smooth at any axis meeting that part of the Cauchy horizon.

For each kind of singularity there are both future-marginally-trapped and past-marginallytrapped cylinders on $\sigma$. This follows from an analysis of the implicit solution (39), but can be seen just by considering asymptotic forms near the different kinds of singularity as they have already occurred. The global picture is complicated by the presence of both past and future Cauchy horizons, but the existence of the exterior in the whole domain of dependence follows as before if $r_{0} \leq \pi / 2$ and follows near to $\sigma$ if $r_{0}>\pi / 2$.

\section{A Non-diagonal Example}

This case is much harder and we have only partial results. In principle, one could repeat the work of the previous section with the non-diagonal interior metrics of Bianchi types II, VIII and IX matched to non-diagonal exterior vacuum metrics. This is hampered by two things: the non-existence, in the literature at least, of even implicit solutions for the interior metric; and the fact that the EFEs are now coupled and cannot be solved one by one.

For the interior solution, the Bianchi type II has been taken furthest and we shall consider just some particular instances of this case. The constants in (4) are $\epsilon=k=0$ and $n=1$ so that the matching conditions from (16)-(23) are

$$
\begin{array}{rll}
\psi & \stackrel{\sigma}{=} & \log a \\
R & \stackrel{\sigma}{=} & a b r_{0} \\
W & \stackrel{\sigma}{=} & r_{0}^{2} / 2 \\
\psi_{\rho} & \stackrel{\sigma}{=} & 0 \\
R_{\rho} & \stackrel{\sigma}{=} & a \\
W_{\rho} & \stackrel{\sigma}{=} & r_{0} / b
\end{array}
$$


together, as always, with $\gamma \stackrel{\sigma}{=} \psi$ and $\gamma_{\rho} \stackrel{\sigma}{=} \psi_{\rho}$.

The field equations for the interior metric are given by (41). They have been solved in terms of $\mu$ subject to a single ODE by Maartens and Nel [6] as follows:

$$
\begin{aligned}
& a(t)=\mu \exp \left(-2 \int^{t} \mu(s)(\alpha-s) d s\right) \\
& b(t)=\mu^{-1} \exp \left(\int^{t} \mu(s)(\alpha-s) d s\right)
\end{aligned}
$$

where $\mu(t)$ satisfies

$$
\mu_{t t}-\frac{4}{\mu}\left(\mu_{t}\right)^{2}+8(\alpha-t) \mu \mu_{t}-\frac{1}{2} \mu^{2}-6(\alpha-t)^{2} \mu^{3}=0 .
$$

In (31), $\alpha$ is an additive constant and the singularity will be at $t=\alpha$. It is possible to reduce the order of the equation for $\mu$, but it does not seem possible to find a general solution. Two particular solutions are given by $\mu(t)=k /(\alpha-t)^{2}$ with $k=5 / 4$ or $k=4 / 3$, and now $a$ and $b$ are just powers of $(\alpha-t)$.

Proceeding as before, we find that $R$ is strictly positive, and in fact analytic, in the domain of dependence. There are trapped surfaces on $\sigma$ between the singularity at $T=\alpha$ and a value $T_{0}$ which depends on the constants of integration. Thus there are trapped surfaces in the exterior.

To obtain the other functions appearing in the exterior metric we need to solve the system of equations (32)-(37). This is a much-studied system and a good deal is known. Local existence in a closely related setting was shown by Chrusciel [2]. To obtain a more global result, and in particular existence out to the Cauchy horizon, the methods of Berger et al. [1] would be expected to work, but we have not done this.

\section{Properties of the solutions}

We shall concentrate on the diagonal cases, where existence is known. We have found that a radiating exterior can be matched to a collapsing interior but there are always trapped surfaces in the exterior. From the work of Berger et al. [1] this means that the solutions cannot be asymptotically flat, so that we have the following corollary to Theorem 1:

Corollary 1 Asymptotically flat vacuum cylindrically symmetric spacetimes cannot be matched to the boundary of solid cylinders in LRS spatially homogeneous perfect fluid spacetimes with diagonal metrics, preserving the cylindrical symmetry.

We now want to present some evidence that these solutions are actually singular on the Cauchy horizon $\mathcal{H}$. We begin by introducing the quantity $u$ defined by

$$
u=R^{1 / 2} \psi
$$

then (34) becomes

$$
u_{x y}=\frac{R_{x} R_{y}}{4 R^{2}} u \text {. }
$$

It follows from this equation that if $R_{x}=0, R \neq 0$ at some value $x=x_{0}$ then $u_{x}$ is constant on $x=x_{0}$, and therefore so is $R^{1 / 2} \psi_{x}$. In the FLRW case, precisely this happens on $\mathcal{H}$ so that $R^{1 / 2} \psi_{x}$ is constant on $\mathcal{H}$. However this quantity diverges in the corner where $\mathcal{H}$ meets the matching surface, and it is therefore singular on $\mathcal{H}$. Once $\psi$ is not differentiable at $\mathcal{H}$, there is no reason to expect $\gamma$ to even be finite there, in which case the metric is singular. 
One can alternatively look at curvature components, specifically at the Weyl spinor in the exterior. We introduce the null tetrad

$$
\begin{aligned}
l & =2^{-1 / 2} \mathrm{e}^{\psi-\gamma}\left(\partial_{T}+\partial_{\rho}\right) \\
n & =2^{-1 / 2} \mathrm{e}^{\psi-\gamma}\left(\partial_{T}-\partial_{\rho}\right) \\
m & =2^{-1 / 2}\left(\mathrm{e}^{-\psi} \partial_{z}+\mathrm{ie}^{\psi} \partial_{\phi}\right)
\end{aligned}
$$

and calculate the (nonzero) components of the Weyl spinor in this tetrad as

$$
\begin{aligned}
& \Psi_{0}=-2 \mathrm{e}^{2(\psi-\gamma)}\left(\psi_{x x}+\frac{1}{R} R_{x} \psi_{x}-\frac{1}{2 R} R_{x x}\right) \\
& \Psi_{2}=2 \mathrm{e}^{2(\psi-\gamma)}\left(\psi_{x y}-\gamma_{x y}\right) \\
& \Psi_{4}=-2 \mathrm{e}^{2(\psi-\gamma)}\left(\psi_{y y}+\frac{1}{R} R_{y} \psi_{y}-\frac{1}{2 R} R_{y y}\right) .
\end{aligned}
$$

If we restrict these quantities to $\sigma$ for the case of an FLRW interior, then we find that $\Psi_{0}=$ $\Psi_{4}=0$ but that $\Psi_{2}$ diverges as the singularity is approached. This is in the corner where $\mathcal{H}$ meets $\sigma$ and then we expect this singularity to propagate along the Cauchy horizon.

We also note the appearance of the term $R_{x x}$ in $\Psi_{0}$, which blows up at $\mathcal{H}$ for all our examples. We cannot explicitly evaluate the other terms in $\Psi_{0}$, but this suggests that $\Psi_{0}$ diverges at $\mathcal{H}$. This component of the Weyl spinor, in this tetrad, is interpreted as incoming radiation, which therefore becomes singular at the Cauchy horizon. This interpretation leads us to a physical explanation of the exterior singularity: suppose at a particular instant we set up Cauchy data with a spatially homogeneous interior, and we want the interior to remain spatially homogeneous even as it collapses. This requires incoming radiation and these examples show that it requires more and more as the interior approaches the singularity, leading to a singularity in the exterior on the Cauchy horizon.

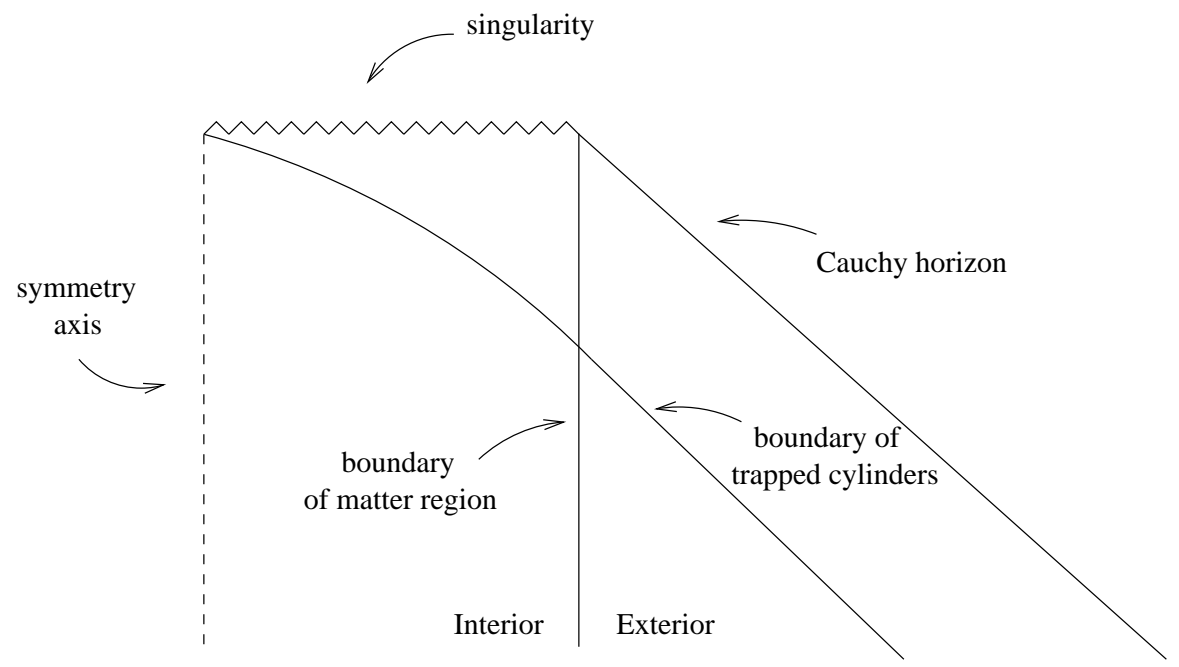

Figure 1: Schematic diagram of the spacetime structure.

\section{Acknowledgments}

FCM thanks Raül Vera and Brien Nolan for usefull discussions, Fundação Calouste Gulbenkian for grant 21-58348-B and Centro de Matemática, Universidade do Minho, for support. 


\section{Appendix}

The EFEs for the cylindrically symmetric vacuum metric (7) are

$$
\begin{aligned}
0 & =\gamma_{T T}-\gamma_{\rho \rho}-\psi_{\rho}^{2}+\psi_{T}^{2}-\frac{e^{4 \gamma}}{4 R^{2}}\left(\left(W_{T}\right)^{2}-\left(W_{\rho}\right)^{2}\right) \\
0 & =R_{T T}-R_{\rho \rho} \\
0 & =\psi_{T T}+\frac{R_{T}}{R} \psi_{T}-\psi_{\rho \rho}-\frac{R_{\rho}}{R} \psi_{\rho}-\frac{e^{4 \gamma}}{2 R^{2}}\left(\left(W_{T}\right)^{2}-\left(W_{\rho}\right)^{2}\right) \\
0 & =W_{T T}-W_{\rho \rho}-\frac{R_{T}}{R} W_{T}+\frac{R_{\rho}}{R} W_{\rho}+4 W_{T} \gamma_{T}-4 W_{\rho} \gamma_{\rho}
\end{aligned}
$$

together with the two constraint equations

$$
\begin{aligned}
\gamma_{\rho} & =\frac{1}{R_{\rho}^{2}-R_{T}^{2}}\left(R R_{\rho}\left(\psi_{T}^{2}+\psi_{\rho}^{2}\right)-2 R R_{T} \psi_{T} \psi_{\rho}+R_{\rho} R_{\rho \rho}-R_{T} R_{T \rho}\right) \\
\gamma_{T} & =\frac{1}{R_{T}^{2}-R_{\rho}^{2}}\left(R R_{t}\left(\psi_{T}^{2}+\psi_{\rho}^{2}\right)-2 R R_{\rho} \psi_{T} \psi_{\rho}+R_{T} R_{\rho \rho}-R_{\rho} R_{T \rho}\right) .
\end{aligned}
$$

The EFEs for LRS spatially homogeneous diagonal dust metrics (4) are

$$
\begin{aligned}
\mu & =2 \frac{a_{t} b_{t}}{a b}+\frac{b_{t}^{2}}{b^{2}}+\frac{k}{b^{2}} \\
0 & =2 \frac{b_{t t}}{b}+\frac{b_{t}^{2}}{b^{2}}+\frac{k}{b^{2}} \\
0 & =\frac{b_{t t}}{b}+\frac{a_{t} b_{t}}{a b}+\frac{a_{t t}}{a}
\end{aligned}
$$

The solution for $k=1$ (Bianchi III) in parametric form is [9]

$$
\begin{aligned}
a \cos \eta & =M(\eta \sin \eta+\cos \eta)+C_{0} \sin \eta \\
b & =C_{1} \cos ^{2} \eta, \quad d t=2 b d \eta
\end{aligned}
$$

where $M$ and $C_{1}$ are positive constants.

The solution for $k=-1$ (Kantowski-Sachs) in parametric form is [9]

$$
\begin{aligned}
a \sinh \eta & =\left[M(\eta \cosh \eta-\sinh \eta)+C_{0} \cosh \eta\right] \\
b & =C_{1} \cosh ^{2} \eta, \quad d t=2 b d \eta
\end{aligned}
$$

with $M$ and $C_{1}$ positive constants.

The EFEs for LRS spatially homogeneous non-diagonal dust metrics (4) of Bianchi II are

$$
\begin{aligned}
\mu & =\frac{b_{t}^{2}}{b^{2}}+2 \frac{a_{t} b_{t}}{a b}-\frac{a^{2}}{4 b^{4}} \\
0 & =2 \frac{b_{t t}}{b}+\frac{b_{t}^{2}}{b^{2}}-\frac{3 a^{2}}{4 b^{4}} \\
0 & =\frac{b_{t t}}{b}+\frac{a_{t t}}{a}+\frac{a_{t} b_{t}}{a b}+\frac{a^{2}}{4 b^{4}}
\end{aligned}
$$

from which the solution of Maartens and Nel [6] given in the text can be found. 


\section{References}

[1] Berger B K, Chrusciel P T \& Moncrief V, Ann. Phys. 237 (1995) 322

[2] Chrusciel P T, Ann. Phys. 202 (1990) 100

[3] Clarke C J S \& Dray T, Class. Quant. Grav. 4 (1987) 265

[4] Israel W, Nuovo Cimento 44 (1966) 1

[5] Kantowski R \& Sachs R K, J. Math. Phys. 7 (1966) 443

[6] Maartens R \& Nel S D, Commun. Math. Phys. 59 (1978) 273

[7] Mars M \& Senovilla J M M, Class. Quant. Grav. 10 (1993) 1865

[8] Mena F C, Tavakol R \& Vera R, Phys. Rev. D66 (2002) 044004

[9] Stephani H, Kramer D, MacCallum M, Hoenselaers C \& Herlt E, Exact solutions of Einstein's field equations, 2nd edition, (Cambridge: Cambridge University Press, 2003)

[10] Taylor M E, Partial differential equations: basic theory, (Berlin: Springer, 1996)

[11] Vera R, Class. Quant. Grav. 19 (2002) 5249 